\title{
EFL Como Requisito Para Estudios De Posgrado En Mexico: El Caso De Los Egresados Del Área De Ciencias Biológico Agropecuarias y Pesqueras de la Universidad Autónoma de Nayarit
}

\author{
M. A. en ELT María Georgina Ochoa García \\ M. A. en ELT Marcela García Ramos \\ Dr. María del Carmen Hernández Cueto \\ M. A. Yun Sang Cheol
}

Universidad Autónoma de Nayarit, Mexico

Doi:10.19044/esj.2018.v14n28p101 URL:http://dx.doi.org/10.19044/esj.2018.v14n28p101

\begin{abstract}
The aim of this study is to get a general overview of the relevance of English as a Foreign Language (EFL) requirement for postgraduate studies in Mexico, since such requirement has been established as one of the main elements of National Quality Postgraduate Programs (PNPC) registered in the National Bureau of Science and Technology (CONACYT) catalog. There are 2,155 programs to be found in such catalog, where 130 of them are cataloged as programs of International Competency; are public university graduates ready to cope with the EFL requirement established in PNPC programs? This research project took a closer look into a group of Mexican university graduates' EFL proficiency levels fulfilled as both: as a fulfillment bachelor's degree requirement, and as an entry requirement to be accepted in a biological and agropecuary sciences masters' degree program from the PCPN.
\end{abstract}

Keywords: English, Foreign Language, EFL, postgraduate requirement.

\section{Resumen}

El propósito de este estudio es obtener un panorama general de la importancia del idioma Inglés como Lengua Extranjera (EFL) como requisito de ingreso para estudios de posgrado en México. En la actualidad, este aspecto ha sido establecido como requisito esencial para pertenecer al Padrón Nacional de Posgrados de Calidad (PNPC) en México del Consejo Nacional de Ciencia y Tecnología (CONACYT). El PNPC cuenta hasta la fecha con 2,155 programas; 130 de ellos pertenecen a la rama de programas de Competencia Internacional; ¿alcanzan los egresados de la universidad pública el requisito de 
EFL establecido en lis programas del PNPC? Este estudio observo de manera directa los niveles de eficiencia en EFL, tanto como de requisito de egreso de los programas de licenciatura de una universidad mexicana, como de ingreso a un programa de maestría en el área de ciencias biológico agropecuarias del PNCP.

Palabras clave: Inglés como lengua Extranjera, EFL, requisito de posgrado

\section{Introducción:}

La creación de programas de estudios de posgrado de calidad, a nivel nacional e internacional, representa una acción estratégica que abona de manera directa a los indicadores del desarrollo de la tercera Meta Nacional del Plan Nacional de Desarrollo (PND) 2013-2018, México con Educación de Calidad, la cual sustenta a la educación superior del país sobre los pilares del desarrollo científico y tecnológico, así como de la innovación, el progreso económico y social sostenible del país (SEP, CONACYT, 2017). Los programas de posgrado de calidad, responden a la necesidad de incrementar y mantener la calidad de la formación ofrecida por los programas de posgrado en el país, mediante la acreditación de los mismos en el Programa Nacional de Posgrados de Calidad (PNPC)..." (PND, 2013 p. 128).

En este sentido, en México, el Consejo Nacional de Ciencia y Tecnología (CONACYT) lanza anualmente una convocatoria para el registro y la evaluación que los programas, nuevos o ya existentes en el padrón de calidad, sean acreditados o re-acreditados como tales. Incluyendo, además, en dicho padrón de calidad, programas de posgrado con nuevas modalidades respondiendo a las necesidades de inclusión, movilidad, flexibilidad e internacionalización de dichos programas educativos. A la fecha, el padrón del PNPC cuenta ya con un total de 2155 programas de posgrado acreditados, en sus diferentes grados, orientaciones, niveles, áreas y modalidades.

\section{Planteamiento del problema}

A pesar de que los estudiantes de los programas de las licenciaturas de la Universidad Autónoma de Nayarit (UAN) han tomado cursos de Inglés como parte de su formación de grado, ya sea de carácter obligatoria o como asignatura de opción libre, los resultados del examen institucional de egreso de Inglés, entre los años 2006 y 2016, muestran que solo el $43 \%$ de los egresados logran aprobar dicho examen; lo que significa que el 57\% de los egresados superan acreditar el Nivel 1, equivalente a la banda A1 de competencias en el idioma Inglés del Marco Común Europeo (CEFR) (Ramos, Hernández, Ochoa $\&$ Cheol 2018). Este hecho sugiere que los estudiantes de los programas del área del CBAP de la UAN con interés en continuar con estudios de maestría no logran cumplir con el requisito de ingreso al programa de maestría del 
CBAP relacionado con el uso del idioma Inglés incluido en el Examen Nacional de Ingreso a Posgrado (EXANI III).

\section{Propósito del estudio}

El presente estudio tiene como objetivo conocer si los estudiantes de los programas de las licenciaturas de Ingeniero Agrónomo, Biólogo, Ingeniero en Pesca y Médico Veterinario Zootecnista, del área de Ciencias Biológico, Agropecuarias y Pesqueras (CBAP) de la UAN logran cumplir con el requisito relacionado con el conocimiento y manejo del idioma Inglés incluido en el Examen Nacional de Ingreso a Posgrado (EXANI III) para el ingreso al programa de maestría en Ciencias Biológico Agropecuarias de la UAN.

\section{Preguntas de investigación}

PI1. ¿Cuál es el nivel de conocimiento y manejo del idioma Inglés de los egresados de los programas de Ingeniero Agrónomo, Licenciado en Biología, Ingeniero en Pesca y Médico Veterinario Zootecnista, del área de Ciencias Biológico, Agropecuarias y Pesqueras del CBAP de la UAN con base a los resultados del examen EXACRI?

PI2. ¿Cuál es el resultado obtenido en el área de Inglés del Examen Nacional de Ingreso a Posgrados (EXANI III) de los candidatos al programa de maestría en Ciencias Biológico Agropecuarias de la UAN, egresados de los programas de Ingeniero Agrónomo, Licenciado en Biología, Ingeniero en Pesca y Médico Veterinario Zootecnista de la UAN?

\section{Antecedentes}

Ante la necesidad educativa de alcanzar estándares internacionales en los ámbitos que conciernen a la educación superior en México. y ante los retos de internacionalización que ésta enfrenta, la Secretaría de Educación Pública (SEP), en el Acuerdo por el que se determinan politicas, estándares, y recomendaciones relacionadas con la enseñanza y certificación de lenguas extranjeras, (SEP, 2006) establece una serie de metas y acciones estratégicas con el objetivo de estandarizar el proceso de enseñanza-aprendizaje del idioma Inglés desde la educación básica hasta la educación superior y de posgrado en el país. Dentro de este documento, la SEP establece, en lo que concierne a la educación superior, que entre los años 2013 y 2018, los egresados de las universidades del país deberían alcanzar el nivel C1 establecido en el Marco Común Europeo de Referencia para las Lenguas: enseñanza, aprendizaje y evaluación (CEFR). Dicho nivel describe al usuario de Inglés como lengua extranjera como: un usuario autónomo o de competencia eficaz. "En general, el usuario avanzado tiene un completo dominio operacional del lenguaje con sólo algunas impropiedades e imprecisiones ocasionales y no sistemáticas. Menores malentendidos pueden presentarse en situaciones no familiares. El 
usuario puede operar argumentaciones detallas y complejas de manera correcta." (SEP, 2006 p.17).

Con base en lo anterior, la Universidad Autónoma de Nayarit, implementa una reforma académica en que incluye un examen del idioma Inglés (EXACRI), para, de acuerdo a Ramos et al. (2018), evaluar las habilidades del idioma Inglés de los estudiantes como requisito de egreso. El EXACRI, de acuerdo a Ramos et al., (2018) contiene 120 reactivos, divididos en tres secciones, estructura, vocabulario y compresión lectora; y clasifica los resultados en los siguientes niveles:

- 41 a 60 puntos: Nivel I (equivalente a la banda A1 del CEFR)

- 61 a 80 puntos: Nivel II (equivalente a la banda A2 del CEFR)

- 81 a 100 puntos: Nivel III (equivalente a la banda B1 del CEFR)

- 101 a 120 puntos: Nivel IV (equivalente a la banda B2 del (CEFR)

A pesar de los esfuerzos institucionales, con relación a la política pública de la mejora continua del proceso de enseñanza-aprendizaje del idioma Inglés como lengua extranjera como parte esencial de la formación de profesionistas, el análisis de la base de datos de los resultados del EXACRI en la cohorte entre la década del 2006 al 2016 presentados por Ramos et al. (2018) muestran que el $57 \%$ de los estudiantes universitarios no alcanzaron a acreditar alguno de los niveles establecidos.

\section{La importancia del idioma Inglés dentro de la educación posgrado}

Dentro de los programas de calidad registrados en el PNPC se encuentran aquellos con orientación a la investigación, los cuales tienen como objetivo lograr que los productos de investigación de los participantes en dichos programas generen conocimiento nuevo, válido y con suficiente calidad, para ser aceptados por la comunidad científica a la que pertenece, tanto para proponer soluciones a problemas del contexto social en el que se desarrollan, como para también ser presentados y/o publicados en los foros y revistas internacionales de su especialidad (CONACYT, 2017). Como se puede observar, estos programas de posgrado de calidad, orientados a la investigación, contribuyen a la formación y el fortalecimiento del capital humano de alto nivel, estrategia 3.5.2 plasmada en el PND 2013-2018. (PND, 2013).

Es en este punto donde radica la importancia de este estudio, ya que el dominio de la competencia lingüística del idioma Inglés como parte de la educación superior representa la piedra la angular para que estos programas de posgrado logren ampliar la cooperación internacional en temas de investigación científica y desarrollo tecnológico, línea de acción establecida dentro del rubro de Educación con Calidad del PND 2013-2018. De igual manera, dentro de los niveles de posgrado que el mismo Consejo Nacional de Ciencia y Tecnología reconoce, se encuentra el nivel de Competencia 
Internacional, descrito como programas que tienen, entre otras características: “... colaboraciones en el ámbito internacional a través de convenios que incluyen la movilidad de estudiantes y profesores, la codirección de tesis y proyectos de investigación conjuntos..." (CONACYT, 2017 p.3). Para este efecto, el CONACYT, dentro del marco referente para la evaluación de programas de posgrado requiere que los mismos programas contemplen mecanismos para impulsar el intercambio académico de estudiantes y profesores, y que a la vez permitan que los estudiantes puedan cursar periodos académicos en otras instituciones nacionales o extranjeras. (CONACYT, 2017).

El padrón de programas de calidad acreditados por el CONACYT cuenta con un total de 2155 programas acreditados de los diferentes grados: especialidad, maestría y doctorado, con orientaciones a la investigación o a la profesionalización, especialidad médica o en posgrado con industria; en las distintas modalidades: escolarizada y no escolarizada. De los cuales, 1,511 son programas de especialidad y maestría, que son aquellos a los que los egresados de los programas de licenciatura pueden ser candidatos de nuevo ingreso. De esos 1,511 programas 130 han sido acreditados con el nivel de competencia internacional, dónde se espera que los egresados de dichos programas sean capaces de colaborar con sus pares en contextos nacionales e internacionales, hecho que solo sería posible a través del uso del idioma Inglés. Ya que, de acuerdo a la Secretaria de Educación (SEP, 2006), en la actualidad, el idioma inglés es el principal lenguaje de los libros, periódicos, aeropuertos, control aéreo, negocios internacionales, conferencias académicas, ciencia y tecnología, diplomacia, deportes, competencias internacionales, música pop y de la publicidad. En materia de informática, agrega la SEP, “...cerca del 80\% de la información que se respalda en las computadoras del mundo se encuentra en inglés y que el $84.3 \%$ de los servidores de Internet del mundo tienen publicada información en inglés." (SEP, 2006 p.12).

\section{Programa de Maestría en Ciencias Biológico Agropecuarias del área del CBAP de la Universidad Autónoma de Nayarit}

El programa de maestría en ciencias biológico agropecuarias de la UAN tiene como objetivo: "Formar Maestros en Ciencias de alto nivel, así como recursos humanos comprometidos en la aplicación y difusión del conocimiento científico en las diferentes opciones de salida con énfasis en el área de atención al medio ambiente para la generación de procesos productivos" (UAN, 2018 p.1).

El plan de estudios de esta maestría se estructura en dos ejes: de formación académica y de investigación. El área de formación académica está diseñada para contribuir al desarrollo de conocimientos básicos y especializados mediante saberes teórico-metodológicos, así como, técnicos e 
instrumentales que contribuyan a la formación integral del estudiante mediante la exploración de distintos contextos y realidades mediante el análisis, contraste y construcción del conocimiento (UAN, 2018).

En el caso específico del conocimiento del idioma Inglés, como parte de este plan de estudios, se encuentra dentro de la convocatoria de ingreso, así como en el perfil de egreso. La convocatoria de ingreso solicita que los candidatos comprueben acreditar un mínimo de 350 puntos en el examen de Inglés como Lengua Extranjera (TOEFL) (UAN; 2018 p.2). Con relación al perfil de egreso, éste establece que el egresado del programa en cuestión tendrá habilidades para presentar resultados de sus investigaciones en foros científicos especializados o de divulgación, así como, para la publicación de los mismos en revistas arbitradas (UAN, 2011); habilidades que sin duda requieren el manejo de competencias comunicativas en el idioma Inglés que le permitan compartir experiencias y conocimientos con sus pares en foros tanto nacionales como internacionales.

Metodología. La presente investigación es un estudio cuantitativo no experimental, de diseño exploratorio transversal ya que éste, tiene la finalidad de obtener información que permita identificar conceptos o variables en un contexto particular para poder establecer las bases para investigaciones futuras (Hernández, Fernández \& Baptista, 2010).

Sujetos. 43 candidatos al programa de maestría en Ciencias Biológico Agropecuarias de la UAN que fueron evaluados entre los ciclos escolares del año 2011 y 2015 egresados de los programas de licenciatura pertenecientes al área del CBAP de la UAN. Distribuidos en los diferentes programas de la siguiente manera: 13 del programa de ingeniero agrónomo, 18 al programa de biología, 4 del programa de ingeniería pesquera y 8 del programa de médico veterinario zootecnista.

Instrumentos. Para este estudio se analizaron primero, los resultados del examen EXACRI obtenidos por los egresados de los programas de licenciatura del área del BAP: Ingeniero Agrónomo (IA), Lic. en Biología (LB), Ingeniero en Pesca (IP) y Médico Veterinario Zootecnista (MVZ) durante los años 2006-2016. Después, fue necesario analizar los resultados en el área de Inglés del Examen Nacional de Ingreso a Posgrado (EXANI III) de los candidatos para ingreso al programa de maestría en ciencias biológico agropecuarias y pesqueras de la UAN. El EXANI III es una de las herramientas utilizadas por las instituciones educativas para la selección de los aspirantes a los programas de posgrado a nivel nacional, este examen permite realizar un diagnóstico con relación a las competencias genéricas necesarias para realizar estudios de posgrado de manera exitosa (CENEVAL, 2013).

El área de Inglés del EXANI III cuenta con 40 reactivos con la intención de "...evaluar la capacidad para reconocer y usar elementos gramaticales que completan con lógica y sentido expresiones y fragmentos y 
conocer el nivel de comprensión lectora en textos especializados escritos en ese idioma." (CENEVAL, 2016 p.12); de los cuales, 20 reactivos para medir la comprensión lectora de dicho idioma, así como con 20 reactivos que miden el uso de la gramática del mismo.

El EXANI III tiene un total de 162 reactivos, y distribuye los resultados de los sustentantes en una curva con forma de campana, donde a la media, los resultados más próximos a 50\% de aciertos se ubican al centro de la campana, situando en los extremos los puntajes muy bajos o muy altos. Es decir, al obtener el $50 \%$ de los aciertos no se interpretaría como haber reprobado el examen, al contrario, significaría haber obtenido una puntuación esperada de acuerdo al diseño del examen; los resultados por cada área, y del examen en general, comienzan en una escala denominada Índice Ceneval, la cual establece 700 puntos como resultado mínimo, y 1300 como resultado máximo, donde se espera que los sustentantes logren alcanzar un puntaje cercano a los 1,000 puntos, media aritmética de del índice establecido (CENEVAL, 2018)

\section{Resultados}

Resultados del examen EXACRI de los egresados de los programas del área del CBAP de la UAN

Los resultados muestran que 1,283 egresados de los cuatro programas en cuestión fueron evaluados durante el periodo de 2006-2016. El 76\% de ellos, 980 egresados, obtuvieron resultados satisfactorios alcanzando entre 41 y 120 puntos, para ubicarse en los cuatro niveles establecidos. Por el contrario, el resto de los examinados, 303, quienes representan el $24 \%$ del total, no lograron alcanzar alguno de los niveles establecidos (véase fig.1).

Fig. 1 Resultados generales del examen EXACRI de los egresados de los programas del CBAP de la UAN periodo 2006-2016.

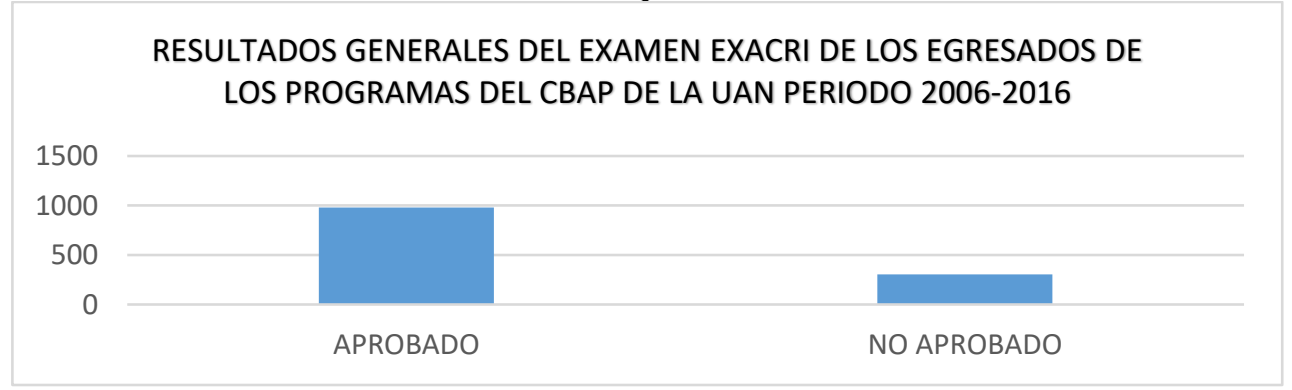

El 49\%, 625 egresados obtuvieron entre 41 y 60 puntos, es decir, el nivel I. Un 20\%, 252 egresados, alcanzaron el nivel II con resultados entre 61 y 80 puntos, un 6\%, 79 egresados, lograron ubicarse en el nivel III con un resultado entre 81 y 100 puntos, mientras que el 2\%, 24 egresados alcanzaron el nivel IV, con resultados superiores a 101 puntos sobre el total de120 que contiene en examen. El resto de los egresados, 303, equivalente al $24 \%$ del 
total, obtuvieron resultados por debajo de 41 puntos, es decir, no lograron alcanzar alguno de los niveles establecidos en el EXACRI, como se puede observar en la fig. 2.

Fig. 2 Resultados EXACRI por niveles de los egresados de los programas del CBAP periodo 2006-2016.

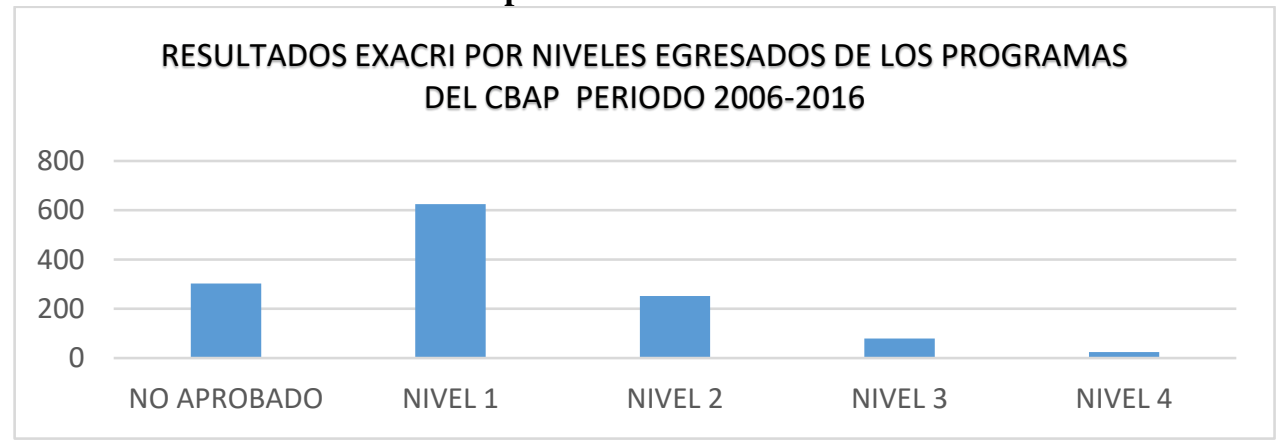

Con relación a los resultados obtenidos dentro de cada uno de los cuatro programas del área del CBAP de la UAN, se observó el siguiente comportamiento: de 435 egresados del programa de Ingeniero Agrónomo, $71 \%, 312$, lograron pasar el requisito establecido, obteniendo los siguientes niveles: 48\%, 210 obtuvieron nivel I; el 17\%, 76 obtuvieron nivel II; el 5\%, 20 egresados alcanzaron nivel III; el 1\%, 6, el nivel IV; por el otro lado, el 28\% de los egresados, 123, no lograron alcanzar alguno de los niveles (véase fig. $3)$.

Fig. 3 Resultados EXACRI del programa de Ing. Agrónomo.

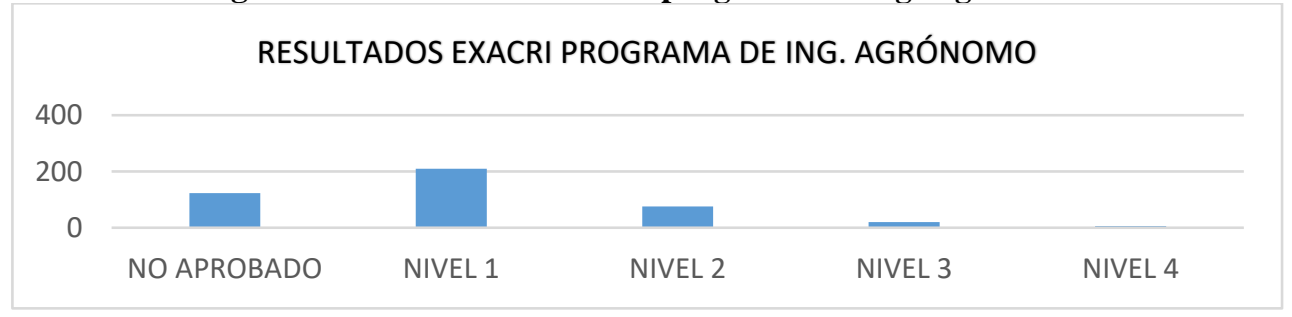

En cuanto al programa de Lic. en Biología, de 203 egresados, el 82\%, 167, logró pasar el requisito establecido obteniendo los siguientes niveles: 48\%, 98, obtuvieron el nivel I; el 21\%, 42, el nivel II; 10\%, 20, nivel III; y el $3 \%, 7$, nivel IV; por otra parte, el1 $8 \%$ restante no lograron alcanzar algún nivel establecido, como se ve en la fig. 4. 
Fig.4 Resultados EXACRI programa de Lic. en Biología.

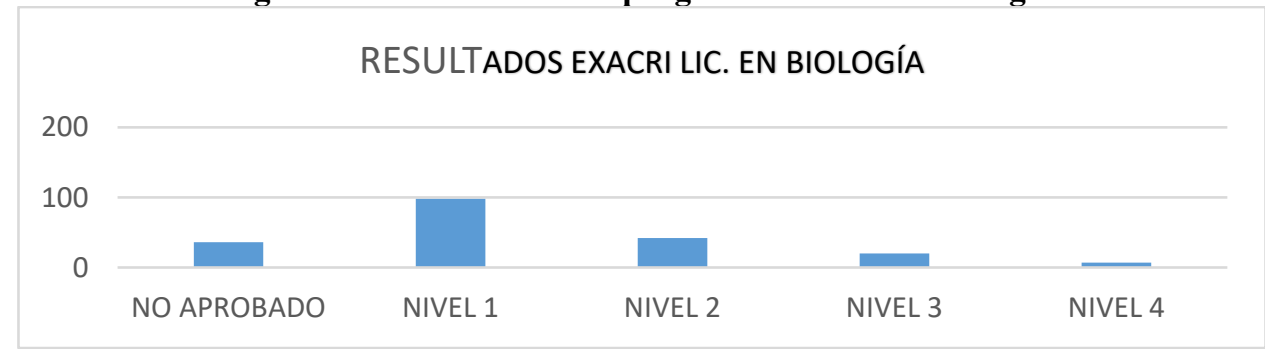

Con relación a los egresados del programa de Ingeniero en Pesca los resultados fueron: el 75\%, 120 egresados lograron alcanzar alguno de los niveles establecidos de la siguiente manera: el 51\% 81 egresados, alcanzaron niel I; 19\%, 31 egresados, nivel II; el 4\%, 6, nivel III; y el 1\%, dos egresados, nivel IV. Por el contrario, el 25\% de los egresados de este programa, 39 de ellos, no lograron acreditar el requisito establecido (véase fig. 5).

Fig. 5 resultados EACRI del programa de Ing. En Pesca.

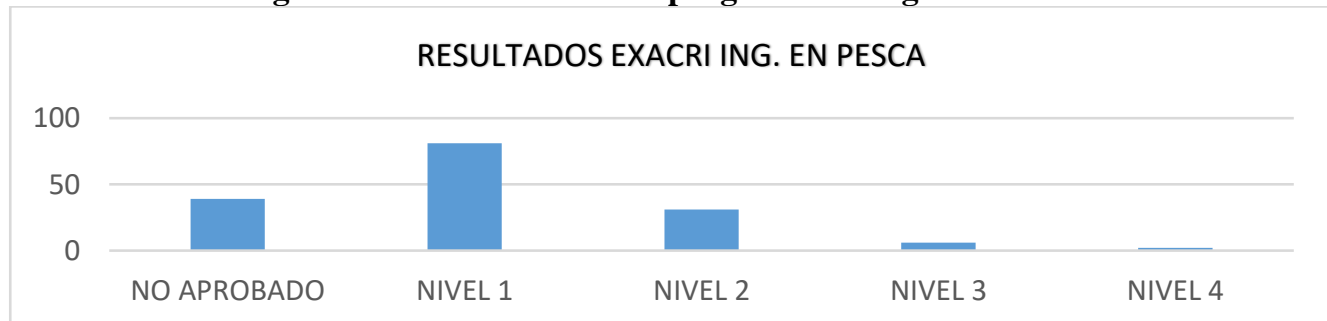

Finalmente, en el caso del programa de Medicina Veterinaria y Zootecnia, de 486 egresados, el 78\%, 381, lograron acreditar el requisito con puntos que los ubicaron en alguno de los cuatro niveles establecidos. Donde se observó que el $49 \%$, 236, obtuvieron resultados dentro del puntaje para nivel I; el resultado del 21\%, 103 egresados, lograron el nivel II; 7\%, 33 egresados, el nivel III y el $2 \%$, nueve egresados, alcanzaron el nivel IV. Por otra parte, el $22 \%$ restante, no alcanzó algún nivel establecido (ver fig. 6).

Fig. 6 Resultados EXACRI del programa de Médico Veterinario Zootecnista.

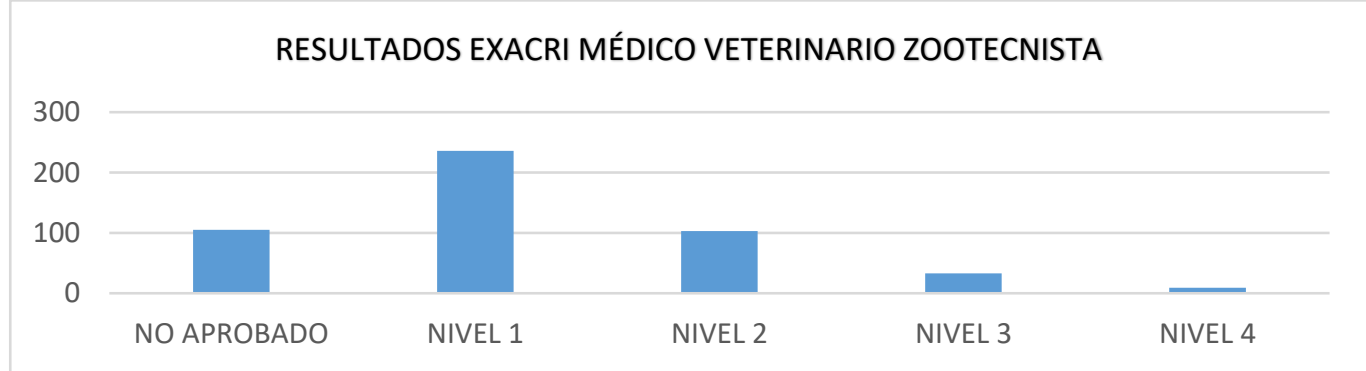


Resultados en el área de Inglés del EXANI III de los candidatos egresados de los programas de licenciatura del área del CBAP de la UAN.

Los datos obtenidos de los resultados en el EXANI III con relación al área de Inglés obtenidos por 43 candidatos al programa de maestría en Ciencias Biológico Agropecuarias de la UAN, egresados de los programas del área de Ciencias Biológico Agropecuarias de la UAN (CBAP), entre el periodo de 2011-2015, indican que el 47\% de los mismos, 20 candidatos, obtuvieron menos de 1000 puntos, mientras que el 53\%, 23, obtuvieron 1000 o más puntos, para promediar una media aritmética de 998.60 puntos en total. Los resultados mencionados previamente se comportaron de la siguiente manera: el $26 \%$ de este segmento, 11 candidatos, se ubican en el rango de 820 a 910 puntos; el 28\%, representado por 12 candidatos, obtuvieron un resultado entre 910 y 1000 puntos; mientras que el 26\%, 11 de ellos, obtuvieron de 1000 a 1090 puntos. El 14\% de los candidatos, 6, obtuvieron puntos en el rango de 1090 a 1180; y finalmente, el registro muestra que el 7\% de estos candidatos, 3, alcanzaron de 1180 a 1270 puntos (ver fig. 7).

Fig. 7 Puntajes en el área de del EXANI II Ingles de los candidatos al programa de maestría en ciencias biológico agropecuarias egresados de los programas del CBAP de la UAN.

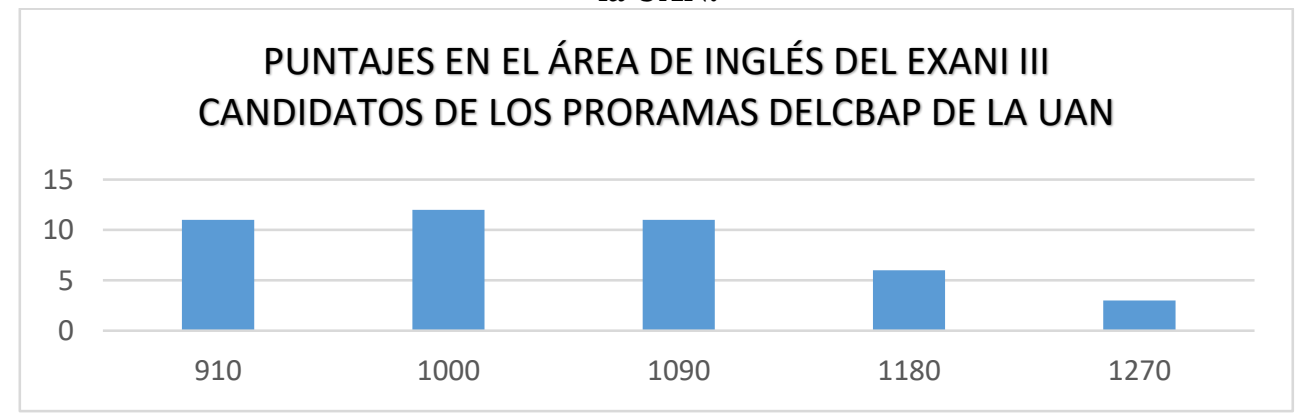

Además, se puede observar que son los candidatos egresados de los programas de Medicina Veterinaria y Zootecnia (MVZ) y de Biología alcanzaron un promedio de 1015 y 1011.67 puntos respectivamente; mientras que los candidatos egresados de los programas de Ingeniero Agrónomo e Ingeniero en Pesca no alcanzaron un promedio de 955 y 983.85 puntos respectivamente, como se puede observar en la FIG.

Fig. 8 Promedio de resultados EXANI III área de Inglés de cada uno de los programas del CBAP de la UAN.

PROMEDIO DE RESULTADOS DE CADA PROGRAMA DEL CBAP DE LA UAN

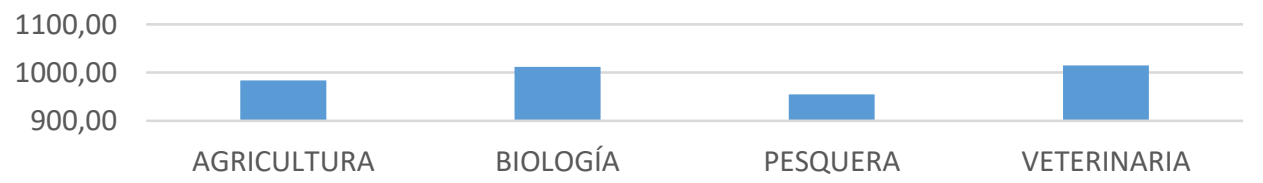




\section{Discusión}

En la actualidad, el uso del idioma Inglés se ha convertido en una herramienta dentro del repertorio de habilidades y competencias que los estudiantes de educación superior, ya que, como lo describe la SEP (2006) “...cerca del $80 \%$ de la información que se respalda en las computadoras del mundo se encuentra en inglés y que el $84.3 \%$ de los servidores de Internet del mundo tienen publicada información en inglés." (SEP, 2006 p.12). Además de que este idioma se ha convertido en el principal lenguaje utilizado dentro del proceso de globalización encontrándose en libros, periódicos, aeropuertos, control aéreo, negocios internacionales, conferencias académicas, ciencia y tecnología, diplomacia, deportes, competencias internacionales, música pop, entre otros aspectos.

Es por eso que, tanto a nivel nacional e internacional, el manejo del idioma en cuestión se ha establecido como un requisito de ingreso a los estudios de posgrado, como es en el caso del programa de la maestría en Ciencias Biológico Agropecuarias de la UAN. En este programa, el requisito de ingreso relacionado con los conocimientos del idioma Inglés y su uso se encuentran establecidos dentro del Examen Nacional de Ingreso a Posgrados (EXANI III). El cual propone como resultado esperado un puntaje cercano a 1000 puntos, media aritmética entre el resultado mínimo de 750 y el resultado máximo de 1300 puntos, descritos dentro del denominado Índice Ceneval.

Por lo anterior, resulta importante conocer la situación académica de los candidatos al programa de la maestría en cuestión, ya sea tanto de los candidatos egresados de los programas pertenecientes a la UAN, para poder así, realizar un análisis que permita proponer acciones a realizar dentro de las áreas de oportunidad detectadas en este estudio.

A continuación, se presentan las respuestas a las preguntas de investigación planteadas con anterioridad.

PI1. ¿Cuál es el nivel de conocimiento y manejo del idioma Inglés de los egresados de los programas de Ingeniero Agrónomo, Licenciado en Biología, Ingeniero en Pesca y Médico Veterinario Zootecnista, del área de Ciencias Biológico, Agropecuarias y Pesqueras del CBAP de la UAN con base a los resultados del examen EXACRI?

De acuerdo con los datos analizados, se puede observar que alrededor del 80 por ciento de los egresados de estos programas logran alcanzar alguno de los niveles establecidos en el examen de egreso EXACRI. Sin embargo, se observa también que la mayoría de estos sólo alcanzan a cubrir los puntajes del nivel I y II; el cuál de acuerdo con lo establecido en el documento Acuerdo por el que se determinan políticas, estándares, y recomendaciones relacionadas con la enseñanza y certificación de lenguas extranjeras, (SEP, 2006), los ubica en las Banda A1 y A2 del CEFR la primera describe como Usuario Elemental, el cual "tiene una competencia básica limitada a situaciones familiares. El 
usuario tiene frecuentes problemas en comprensión y expresión. El usuario no es capaz de utilizar lenguaje complejo" (SEP, 2006, p.19).; y la segunda, describe al Usuario Modesto: "cuenta con habilidades básicas del lenguaje... Los usuarios de este nivel son capaces de entender los puntos principales de textos simples, pueden comunicarse en la mayoría de las situaciones familiares, y pueden entender avisos breves y estandarizados, así como direcciones orales breves. (SEP, 2006, p.18). Esto significa que esta gran cantidad de egresados de estos programas podrían encontrar mayores dificultados relacionadas con el manejo y uso del idioma Inglés como requisito de ingreso a estudios de posgrado, ya que es aquí donde serían requeridos de un mayor repertorio de habilidades lingüísticas del idioma en cuestión.

Por otro lado, se puede observar que, en menor cantidad, alrededor del 10 por ciento de los egresados, lograron alcanzar los niveles III y IV de EXACRI, los cuales podrían equipararse con los usuarios de la Banda B1 denominado Usuario Intermedio, los cuales "...tienen la capacidad de enfrentar situaciones que requieren un uso predecible del lenguaje, pueden leer libros de texto y artículos simples, así como escribir cartas personales sencillas, o realizar notas durante una reunión. Los usuarios comprenden un amplio vocabulario y son capaces de utilizar estilos apropiados de comunicación para una variedad de situaciones" (SEP, 2006, P.18)

PI2. ¿Cuál es el resultado obtenido en el área de Inglés del Examen Nacional de Ingreso a Posgrados (EXANI III) de los candidatos al programa de maestría en Ciencias Biológico Agropecuarias de la UAN, egresados de los programas de Ingeniero Agrónomo, Licenciado en Biología, ¿Ingeniero en Pesca y Médico Veterinario Zootecnista de la UAN?

Los resultados analizados muestran que en general, la mayoría de los candidatos a este programa de maestría, lograron superar el puntaje esperado de 1000 puntos en el área de Inglés del EXANI III, establecido dentro del Índice Ceneval establecido, siendo menor el número de candidatos que se alejaron del resultado esperado. También se puede observar que el promedio general de los resultados obtenidos, solo queda a menos de dos puntos por debajo de la media establecida. Esto significa que los candidatos al programa de maestría, independientemente del programa de licenciatura del área del CBAP al que pertenezcan, cumplen con el requisito de ingreso a estudios de posgrado establecido para los programas de posgrado pertenecientes al padrón de calidad nacional (PNPC).

\section{Conclusion}

La importancia del uso del idioma Inglés alrededor del mundo en la actualidad, radica en el hecho que, como Harmer (2007) lo explica, desde finales del siglo XX este idioma se perfilaba hacia convertirse en una genuina 
lengua franca, es decir, convertirse en el idioma ampliamente usado como medio de comunicación entre personas que no comparten dicho idioma como lengua materna. Entre los factores que este autor enumera como posibles factores para que este fenómeno extensión del idioma Inglés se lleve a cabo: un movimiento histórico de colonización, comercio global, turismo, popularización de la cultura, así como el intercambio de información. En este sentido, Harmer (2007) explica que la gran parte del discurso académico alrededor del mundo se lleva a cabo en el idioma Inglés; ya que es éste el la lengua franca usada en conferencias así como en revistas de artículos científicos en diversas áreas de estudio; de igual manera agrega, que el canal más grande de comunicación como lo es el internet, ha sido dominado por el uso de este Idioma. Por su parte, la Secretaría de Educación en México, ha expresado también que "....cerca del $80 \%$ de la información que se respalda en las computadoras del mundo se encuentra en inglés y que el $84.3 \%$ de los servidores de Internet del mundo tienen publicada información en inglés." (SEP, 2006 p.12).

En este sentido, el presente estudio permite obtener un panorama general del nivel de conocimiento y uso del idioma Inglés como requisito de egreso de los programas de educación superior del área de las ciencias biológico agropecuarias y pesqueras de la UAN. Donde se puede concluir que la mayoría de los egresados de dichos programas pueden cumplir con un requisito de conocimiento y uso del idioma Inglés establecido de manera institucional, y que, además, son capaces de acercarse a la media establecida como requisito de ingreso a los programas de calidad de estudios de posgrado a nivel nacional. Además, se pudo observar que los candidatos al programa de maestría en cuestión egresados de los programas de Lic. en Biología y de Médico Veterinario Zootecnista lograron acercarse más, o superar el resultado esperado como requisito establecido en el examen nacional de ingreso a estudios de posgrado. Dato interesante que invita a realizar un análisis más profundo en búsqueda de alguna explicación a este fenómeno.

Este estudio, puede a su vez, servir como base para futuros estudios relacionados con el manejo y uso del idioma Inglés como parte de la currícula de estudios a nivel superior; así como del requisito de dicho idioma como requisito de estudios de posgrado a nivel internacional. Para así contribuir al establecimiento y mejora de la política pública con relación a la importancia de los procesos de enseñanza, aprendizaje y evaluación del dominio de este idioma a nivel nacional e internacional.

\section{References:}

1. CENEVAL. (2016). Guía EXANI III Examen Nacional de Ingreso al Posgrado Disponible en http://www.ceneval.edu.mx/documents/20182/36170/Gu\%C3\%ADa+ 
EXANI-III+14a+ed+Final.pdf/449ccecd-516f-45d0-9b7028d9918d272a

2. CENEVAL. (2018). Guía EXANI III Examen Nacional de Ingreso al Posgrado. MÉXICO: CENEVAL.

3. CONACyT. (ABRIL de 2015). Anexo A. Programas de orientación a la investigación. CONACyT . Disponible en https://www.conacyt.gob.mx/index.php/becas-y-posgrados/programanacional-de-posgrados-de-calidad/convocatorias-avisos-y-resultados

4. CONACYT. (2017). Marco de referencia para la evaluación y seguimiento de programas de posgrado de calidad. MÉXICO: CONACYT.

5. Gobierno de la República. Plan Nacional de Desarrollo 2013-2018 Obtenido de http://pnd.gob.mx/

6. Harmer, J. (2007). The practice of English language teachig. England: Pearson.

7. Hernández Sampieri, R., Fernández Collado, C., \& Baptista Lucio, P. (2010). Metodología de la investigación. Méxcio D.F.: McGraw-Hill.

8. Ramos, M., Hernández, M. C., Ochoa, G., \& Cheol, Y. S. (2018). An overview of a Public Mexican University. U.S. China Education Review, 27-32.

9. Secretaria de Educación Pública (SEP). (2006). Acuerdo por el que se determinan políticas, estándares, y recomendaciones realcionadas con la enseñanza, y certificación de enguas extranjeras, así como del Espñol como lengua adicional. México: SEP.

10. Universidad Autónoma de Nayarit. (14 de Junio de 2018). Estudio de Pertinencia.Universidad Autónoma de Nayarit. Obtenido de www.uan.edu.mx/maestria-en-ciecnias-biológicoagropecuarias\#inicio 